\title{
SUSY on the lattice
}

\author{
I. Montvay ${ }^{a}$ \\ ${ }^{a}$ Deutsches Elektronen Synchrotron DESY, \\ Notkestr. 85, D-22603 Hamburg, Germany
}

The motivation and perspectives of numerical simulations of supersymmetric Yang-Mills theories are reviewed.

\section{INTRODUCTION}

Supersymmetric quantum field theories, reviewed for instance in refs. [1]-[3], have peculiar properties. They represent an interesting special subset of quantum field theories. Because of their highly symmetric nature, they are best suited for analytical studies, which sometimes lead to exact solutions [4].

\subsection{Supersymmetric Yang-Mills theory}

Since local gauge symmetries play a very important rôle in Nature, there is a particular interest in supersymmetric gauge theories. The simplest examples are supersymmetric Yang-Mills (SYM) theories which are supersymmetric extensions of pure gauge theories. Depending on the number $N$ of supersymmetry generator pairs $Q_{i \alpha}, \bar{Q}_{i \dot{\alpha}},(i=1,2, \ldots, N)$, one speaks about $N=1$ "simple" supersymmetry or $N \geq 2$ "extended" supersymmetry. Interesting examples of SYM theories have, for instance, $N=1$ or 2 supersymmetry.

The SYM action with $N=1$ supersymmetry can be written as follows:

$$
\begin{aligned}
S_{S Y M}= & \frac{1}{4 \pi} \operatorname{Im}\left\{\tau \int d^{4} x d^{2} \theta \operatorname{Tr}\left(W^{\alpha} W_{\alpha}\right)\right\} \\
= & \frac{1}{g^{2}} \int d^{4} x \operatorname{Tr}\left[-\frac{1}{2} F_{\mu \nu} F^{\mu \nu}\right. \\
& \left.-i \lambda \sigma^{\mu}\left(D_{\mu} \bar{\lambda}\right)+i\left(D_{\mu} \bar{\lambda}\right) \bar{\sigma}^{\mu} \lambda+D^{2}\right] \\
& +\frac{\Theta}{16 \pi^{2}} \int d^{4} x \operatorname{Tr}\left[F_{\mu \nu} \tilde{F}^{\mu \nu}\right] .
\end{aligned}
$$

Here $\tau$ is a complex coupling parameter

$\tau \equiv \frac{\Theta}{2 \pi}+\frac{4 \pi i}{g^{2}}$ combining the gauge coupling $g$ with the $\Theta$ parameter. The first line of eq. (1) is in superfield notation, in terms of the spinorial field strength superfield $W(x, \theta, \bar{\theta})_{\alpha}$ which depends on the fourcoordinate $x$ and the anticommuting Weyl-spinor variables $\theta_{\alpha}, \bar{\theta}_{\dot{\alpha}}(\alpha, \dot{\alpha}=1,2)$. After performing the grassmanian integration on $\theta$, one obtains the second form of the SYM action in eq. (11) in terms of the component fields: the field strength tensor $F_{\mu \nu}$, its dual $\tilde{F}_{\mu \nu} \equiv \frac{1}{2} \epsilon_{\mu \nu \rho \sigma} F_{\rho \sigma}$, the Majorana fermion field in the adjoint representation $\lambda, \bar{\lambda}$ and the auxiliary field $D$. Of course, $D_{\mu}$ denotes gauge covariant derivative and $\sigma^{\mu} \equiv\left\{1, \sigma^{1,2,3}\right\}$. (For details of the formalism see refs. [1]-[3].)

Since the auxiliary field without kinetic term $D$ can be trivially integrated out, $S_{S Y M}$ is nothing else but a Yang-Mills theory with a massless Majorana fermion in the adjoint representation. Hence the Yang-Mills theory of such a field, called "gaugino" or, in the context of strong interactions, "gluino", is automatically supersymmetric. Introducing a non-zero gaugino mass $m_{\tilde{g}}$ breaks supersymmetry "softly". Such a mass term is:

$m_{\tilde{g}}\left(\lambda^{\alpha} \lambda_{\alpha}+\bar{\lambda}^{\dot{\alpha}} \bar{\lambda}_{\dot{\alpha}}\right)=m_{\tilde{g}}(\bar{\Psi} \Psi)$.

Here in the first form the Majorana-Weyl components $\lambda, \bar{\lambda}$ are used, in the second form the DiracMajorana field $\Psi$.

$S_{S Y M}$ in eq. (11) defines the simplest, $N=1$ SYM theory. It is reasonable to start the nonperturbative lattice studies by this. An important feature of this theory is its global $U(1)_{\lambda}$ chiral symmetry which coincides with the so called $R$-symmetry generated by the transformations

$\theta_{\alpha}^{\prime}=e^{i \varphi} \theta_{\alpha}, \quad \bar{\theta}_{\dot{\alpha}}^{\prime}=e^{-i \varphi} \bar{\theta}_{\dot{\alpha}}$. 
This is equivalent to

$\lambda_{\alpha}^{\prime}=e^{i \varphi} \lambda_{\alpha}, \quad \bar{\lambda}_{\dot{\alpha}}^{\prime}=e^{-i \varphi} \bar{\lambda}_{\dot{\alpha}}, \quad \Psi^{\prime}=e^{-i \varphi \gamma_{5}} \Psi$.

The $U(1)_{\lambda}$-symmetry is anomalous: for the corresponding axial current $J_{\mu} \equiv \bar{\Psi} \gamma_{\mu} \gamma_{5} \Psi$, in case of $S U\left(N_{c}\right)$ gauge group with coupling $g$, we have

$\partial^{\mu} J_{\mu}=\frac{N_{c} g^{2}}{32 \pi^{2}} \epsilon^{\mu \nu \rho \sigma} F_{\mu \nu}^{r} F_{\rho \sigma}^{r}$.

However, the anomaly leaves a $Z_{2 N_{c}}$ subgroup of $U(1)_{\lambda}$ unbroken. This can be seen, for instance, by noting that the transformations

$\Psi \rightarrow e^{-i \varphi \gamma_{5}} \Psi, \quad \bar{\Psi} \rightarrow \bar{\Psi} e^{-i \varphi \gamma_{5}}$

are equivalent to

$m_{\tilde{g}} \rightarrow m_{\tilde{g}} e^{-2 i \varphi \gamma_{5}}, \Theta_{S Y M} \rightarrow \Theta_{S Y M}-2 N_{c} \varphi$,

where $\Theta_{S Y M}$ is the $\Theta$-parameter of gauge dynamics. Since $\Theta_{S Y M}$ is periodic with period $2 \pi$, for $m_{\tilde{g}}=0$ the $U(1)_{\lambda}$ symmetry is unbroken if

$\varphi=\varphi_{k} \equiv \frac{k \pi}{N_{c}}, \quad\left(k=0,1, \ldots, 2 N_{c}-1\right)$.

For this statement it is essential that the topological charge is integer.

The discrete global chiral symmetry $Z_{2 N_{c}}$ is expected to be spontaneously broken by the nonzero gaugino condensate $\langle\lambda \lambda\rangle \neq 0$ to $Z_{2}$ defined by $\left\{\varphi_{0}, \varphi_{N_{c}}\right\}(\lambda \rightarrow-\lambda$ is a rotation $)$. Instanton calculations (for a review and references see (5) give at $\Theta_{S Y M}=0$ in $N_{c}$ degenerate vacua $\left(k=0, \ldots, N_{c}-1\right)$

$\frac{g^{2}}{32 \pi^{2}}\langle\lambda \lambda\rangle_{0}=\frac{4 e^{2 \pi i k / N_{c}}}{\left[\left(N_{c}-1\right) !\left(3 N_{c}-1\right)\right]^{1 / N_{c}}} \Lambda_{S Y M}^{3}$

Here $\Lambda_{S Y M}$ denotes the $\Lambda$-parameter of the gauge coupling. The transformation with $\Theta_{S Y M}$ is

$\langle\lambda \lambda\rangle_{\Theta_{S Y M}}=e^{i \Theta_{S Y M} / N_{c}}\langle\lambda \lambda\rangle_{0}$.

In general, an important and interesting question is whether supersymmetry can be broken spontaneously or not. In pure SYM theory, without additional matter supermultiplets, this is not expected to occur. An argument for this is given by the non-zero value of the Witten index [6]

$w \equiv \operatorname{Tr}(-1)^{F}=n_{\text {boson }}-n_{\text {fermion }}$, which is equal to the difference of the number of bosonic minus fermionic states with zero energy. It is supposed not to change with the parameters of the theory. For SYM theory we have $w_{S Y M}=N_{c}$, therefore there is no spontaneous supersymmetry breaking. (The $N_{c}$ ground states discussed above correspond to this Witten index.)

\subsection{Seiberg-Witten solution}

The SYM theory with $N=2$ extended supersymmetry is a highly constrained theory which has, however, more structure than the relatively simple $N=1$ case discussed above. In particular, besides the $N=1$ "vector superfield" containing the gauge boson and gaugino $\left(A_{\mu}, \lambda\right)$, it also involves an $N=1$ "chiral superfield" $\left(\phi, \lambda^{\prime}\right)$ in the adjoint representation which consists of the complex scalar $\phi$ and the Majorana fermion $\lambda^{\prime}$. The Majorana pair $\left(\lambda, \lambda^{\prime}\right)$ can be combined to a Diracfermion $\psi$ and then the vector-like (non-chiral) nature of this theory can be made explicit.

The main new feature of $N=2 \mathrm{SYM}$ is that it contains also scalar fields, hence there is the possibility of Higgs mechanism. Let us here consider only an $S U(2)$ gauge group, when the Higgs mechanism implies the symmetry breaking $S U(2) \rightarrow U(1)$. The complex expectation value of the scalar field $\langle\phi\rangle$ parametrizes the moduli space of zero-energy degenerate vacua. The degeneracy is a consequence of extended supersymmetry. Due to the Higgs mechanism the "charged" gauge bosons become heavy and the low-energy effective theory is $N=2 \mathrm{SYM}$ with $U(1)$ gauge group.

As it has been shown by Seiberg and Witten [4], extended supersymmetry and asymptotic freedom can be used to determine exactly the lowenergy effective action in the Higgs phase, if the vacuum expectation value is large. It turns out that, besides the singularity which corresponds to asymptotic freedom at infinity, at strong couplings there are two singularities of the effective action which describe light monopoles and dyons, respectively.

The unprecedented achievement of an exact solution for a non-trivial four-dimensional quantum field theory induced a considerable activity among theoreticians. Recently there is a new 
way of considering non-perturbative problems in quantum field theory. It is an interesting question how much the more conventional approach based on lattice regularization can contribute to these new insights.

\section{LATTICE FORMULATION}

The simplest supersymmetric gauge theory is $N=1 S U(2)$ SYM. Massive gluinos (Majorana fermions in the adjoint representation) break supersymmetry softly. As discussed in section 1.1. the supersymmetric point is at $m_{\tilde{g}}=0$.

\subsection{Actions}

The Curci-Veneziano action of $N=1 \mathrm{SYM}$ [7] is based on Wilson fermions. The effective gauge action obtained after integrating out the gluino field is given by

$S_{C V}=\beta \sum_{p l}\left(1-\frac{1}{2} \operatorname{Tr} U_{p l}\right)-\frac{1}{2} \log \operatorname{det} Q[U]$,

where the fermion matrix is

$Q_{y v, x u}=\delta_{y x} \delta_{v u}-K \sum_{\mu= \pm} \delta_{y, x+\hat{\mu}}\left(1+\gamma_{\mu}\right) V_{v u, x \mu}$

and the gauge link in the adjoint representation is defined as

$V_{r s, x \mu}=\frac{1}{2} \operatorname{Tr}\left(U_{x \mu}^{\dagger} \tau_{r} U_{x \mu} \tau_{s}\right)$.

The Majorana nature of the gaugino is represented in eq. (13) by the factor $\frac{1}{2}$ in front of $\log \operatorname{det} Q$. There is no problem with the sign of $\operatorname{det} Q$ because we have $\operatorname{det} Q=\operatorname{det} \tilde{Q}$, where

$\tilde{Q} \equiv \gamma_{5} Q=\tilde{Q}^{\dagger}$

is the hermitean fermion matrix. From the relations

$C Q C^{-1}=Q^{T}, \quad B \tilde{Q} B^{-1}=\tilde{Q}^{T}$,

with the charge conjugation matrix $C$ and $B \equiv$ $C \gamma_{5}$, it follows that every eigenvalue of $Q$ and $\tilde{Q}$ is (at least) doubly degenerate. Therefore, with the (real) eigenvalues $\tilde{\lambda}_{i}$ of $\tilde{Q}$, we have

$\operatorname{det} \tilde{Q}=\prod_{i} \tilde{\lambda}_{i}^{2} \geq 0$.
The doubling of the eigenvalues of $\tilde{Q}$ and/or $Q$ implies that for fermions in the adjoint representation zero eigenvalues always occur in pairs, as in the continuum [8]. (This is different for fermions in the fundamental representation: see for instance [9].)

Of course, the lattice action in eq. (13) is not unique. Another possibility is based on fivedimensional domain walls [10]. In this approach one knows the value of the bare fermion mass where the supersymmetric continuum limit is best approached and one may have advantages from the point of view of the speed of symmetry restoration, but one has to pay with the proliferation of (auxiliary) fermion flavours. There are also speculations about direct dimensional reduction on the lattice from $d=10$ or $d=6$ dimensions down to the physically interesting case $d=4$, started by ref. [11] and continued by ref. [12]. However, up to now it has not yet been demonstrated that this approach does really work.

\subsection{Algorithms}

As one can see in the lattice action in eq. (13), Majorana fermions effectively mean a flavour number $N_{f}=\frac{1}{2}$. This can be achieved by the hybrid classical dynamics algorithm [13], which is applicable to any number of flavours. Since it is a finite step size algorithm, an extrapolation to zero step size is needed. This approach has been implemented and successfully tested for the CurciVeneziano action (13) by Donini and Guagnelli in ref. 114.

Another possibility to simulate non-even numbers of flavours is based on the multi-bosonic algorithm [15]. A two-step variant using a noisy correction step [16] has been developed in ref. [17. This algorithm is based, for general $N_{f}$, on the approximation

$\operatorname{det}\left(Q^{\dagger} Q\right)^{N_{f} / 2} \simeq \frac{1}{\operatorname{det} P_{n}\left(Q^{\dagger} Q\right)}$,

where the polynomial $P_{n}$ satisfies

$\lim _{n \rightarrow \infty} P_{n}(x)=x^{-N_{f} / 2}$

within a suitably chosen positive interval $x \in$ $[\epsilon, \lambda]$, which covers the spectrum of $Q^{\dagger} Q$ in typical gauge configurations. In the two-step variant 
one uses a first polynomial $\bar{P}_{\bar{n}}$ for a crude approximation and realizes a fine correction by another polynomial $P_{n}$, where now

$$
\lim _{n \rightarrow \infty} \bar{P}_{\bar{n}}(x) P_{n}(x)=x^{-N_{f} / 2} \text { for } x \in[\epsilon, \lambda] \text {. }
$$

The fermion determinant is approximated as

$$
\operatorname{det}\left(Q^{\dagger} Q\right)^{N_{f} / 2} \simeq \frac{1}{\operatorname{det} \bar{P}_{\bar{n}}\left(Q^{\dagger} Q\right) \operatorname{det} P_{n}\left(Q^{\dagger} Q\right)}
$$

The advantage of this two-step variant is its smaller storage requirement and shorter autocorrelations, because the order of the first polynomial $\bar{n}$ can be kept small. (In praxis, for instance, $\bar{n} \leq 10-12$ for lattice sizes $\leq 8^{3} \cdot 16$.) As a consequence of the smallness of $\bar{n}$, this remains a large step size algorithm, also in the limit of good precision.

In order to achieve an exact algorithm in this two-step variant of the multi-bosonic algorithm, in principle, an extrapolation to $n \rightarrow \infty$ is needed, together with measurement-corrections for eigenvalues outside $[\epsilon, \lambda]$. In praxis, however, $(\bar{n}, n)$ and $[\epsilon, \lambda]$ can be chosen such that these kinds of corrections are smaller than the statistical errors.

Random matrix models suggest that the fluctuations of the minimal eigenvalue are inversely proportional to the lattice volume. (For references and a recent summary see ref. [18].) This is advantageous for the choice of the interval $[\epsilon, \lambda]$. For the calculation of the necessary polynomials procedures written in Maple are available [19. The quality of the polynomial approximations is illustrated by figure 1 .

Besides the above "hermitean" version "complex" (non-hermitean) variants of the multi-bosonic algorithms [20] can also be exploited, preferably also in two-step variants. In order to improve performance, preconditioning according to ref. 21] turned out to be very useful.

\subsection{Quenched computations}

Quenched calculations without the effect of dynamical gluinos can be useful for roughly localizing the critical region in the hopping parameter $K$ and for testing the methods to determine masses. Such calculations have been performed recently in refs. 222 and 23]. An example of the obtained

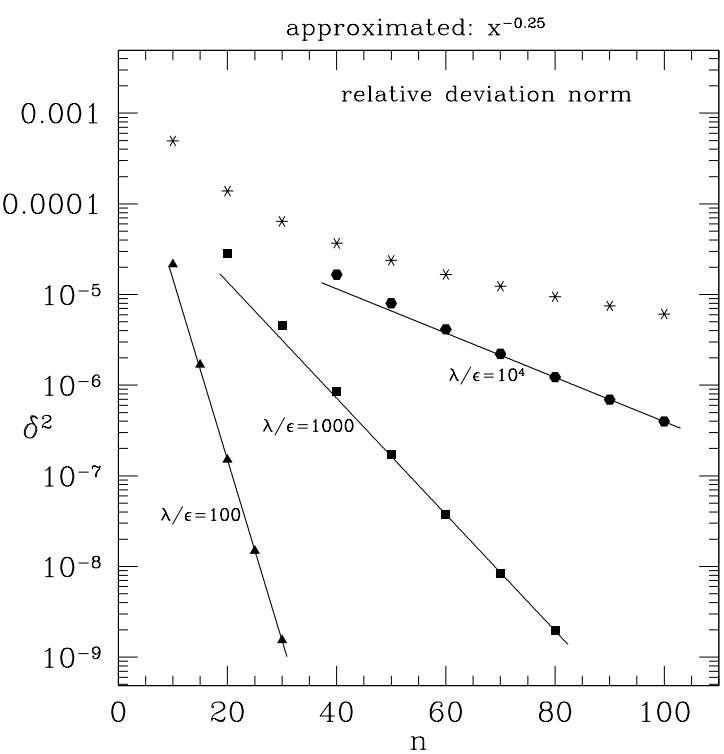

Figure 1. The deviation norm $\delta^{2}$ of the polynomial approximations of $x^{-1 / 4}$ as function of the order for different values of $\lambda / \epsilon$. The asterisks show the $\epsilon / \lambda \rightarrow 0$ limit. (Ref. [19])

lowest masses is shown in figure 2. Notation conventions are as follows: $\tilde{\pi}$ and $\tilde{\sigma}$ denote, respectively, the pion-like and sigma-like states made out of gluinos. $\tilde{\chi}$ is a Majorana fermion state made out of gluons and gluinos.

\section{FIRST RESULTS AND OUTLOOK}

The goal of the DESY-Münster-Athens collaboration [24] is to study non-perturbative properties of SYM theories by Monte Carlo simulations. The computations are performed on the APEQuadrics at DESY-Zeuthen and on the CRAYT3E at HLRZ-Jülich by using the two-step multibosonic fermion algorithm for gluinos discussed in section 2.2 .

\subsection{Chiral symmetry breaking}

An important first step in the numerical investigations is to find the critical hopping parameter $K_{c r}$ corresponding to zero gluino mass. As it is discussed in section 1.1, the supersymmetric point is at zero gluino mass. This tells how the supersymmetric continuum limit $\beta \rightarrow \infty$ has to be performed in the $(\beta, K)$-plane. The basic ex- 


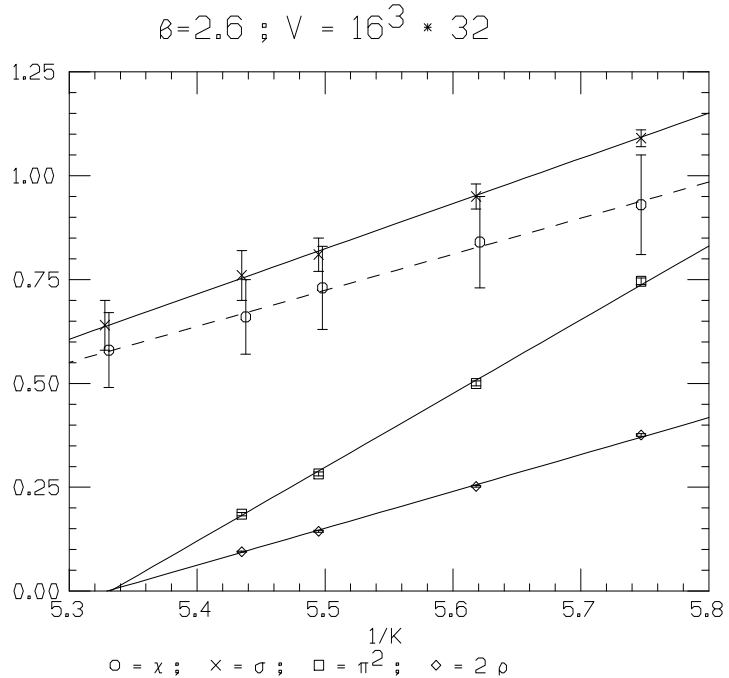

Figure 2. Masses of $\tilde{\pi}, \tilde{\sigma}, \tilde{\chi}$ and $2 \rho$ as a function of $1 / K$ (the mass of $\tilde{\pi}$ is squared). The extrapolation at $K_{c r}$ is also shown (curves). (Ref. [23])

pectation is that at $K=K_{c r}$, for gauge group $S U\left(N_{c}\right)$, there are $N_{c}$ degenerate ground states with different values of the gluino condensate (see eq. (10)). This corresponds to the expected spontaneous global chiral symmetry breaking pattern $Z_{2 N_{c}} \rightarrow Z_{2}$ [6], 25].

The coexistence of several different vacua implies a first order phase transition at $K=K_{c r}$, at least in the continuum limit. At finite lattice spacing in the $(\beta, K)$-plane this might be represented just by cross-over lines. Nevertheless, the simplest possibility is a first order phase transition already for finite $\beta$. For instance, in case of $S U(2)$ gauge group the phase structure may be as shown by figure 3. An alternative phase structure can be motivated by the modified VenezianoYankielowicz effective low energy action proposed recently by Kovner and Shifman [26]. They suggest the existence of an additional massless phase with no chiral symmetry breaking. On the lattice this could lead to a more complicated structure, for instance, because the symmetric phase could be stable or metastable in some intermediate range of the hopping parameter.

An interesting point is the dependence of the phase structure on the gauge group. For example, in case of $S U(3)$ there are at least three degenerate vacua and beyond the critical hopping pa-

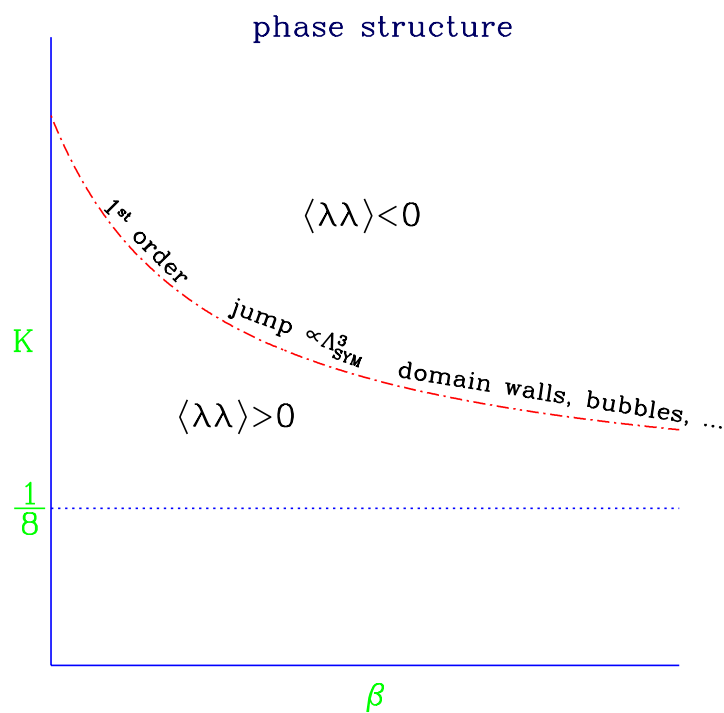

Figure 3. Expected phase structure in the $(\beta, K)$ plane. The dashed-dotted line is a first order phase transition at zero gluino mass.

rameter $K>K_{c r}$ we expect to be at $\Theta_{S Y M}=\pi$.

Since the Monte Carlo simulations are usually done at non-zero gluino mass $m_{\tilde{g}} \neq 0$, the best procedure is to derive analytically the dependence of different quantities on $m_{\tilde{g}}$ [27] and compare them directly with numerical data. In case of the gluino condensate the best possibility is to study the difference $\Delta\langle\lambda \lambda\rangle$ between the values of $\langle\lambda \lambda\rangle$ in different vacua. The advantage is that the additive ultraviolet divergences, due to the breaking of supersymmetry at finite lattice spacing, cancel.

\subsection{Bound state spectrum}

The low energy effective actions predict that at the supersymmetric point the low lying states are organized in massive supermultiplets. The simplest possibility is to have a lowest supermultiplet generated by a superfield made out of gluinos 25]. On the lattice such states are $\tilde{\pi}, \tilde{\sigma}$ and $\tilde{\chi}$ discussed already in section 2.3. Other obvious candidates are glueball states observed in pure Yang-Mills theory, which might also belong to another supermultiplet. Of course, the notion of constituents is in general not expected to work perfectly in a strongly interacting theory. Therefore one has to be open minded towards non-trivial mixings.

One of the main goals of the DESY-Münster- 
Athens collaboration is to determine the masses of low lying states in the vicinity of the supersymmetric point at zero gluino mass. First results are presented at this conference. In general, as shown by figure 1 , the supersymmetric degeneracy of low lying states is not yet observed. Further simulations closer to $K_{c r}$ and at larger $\beta$ are in progress.

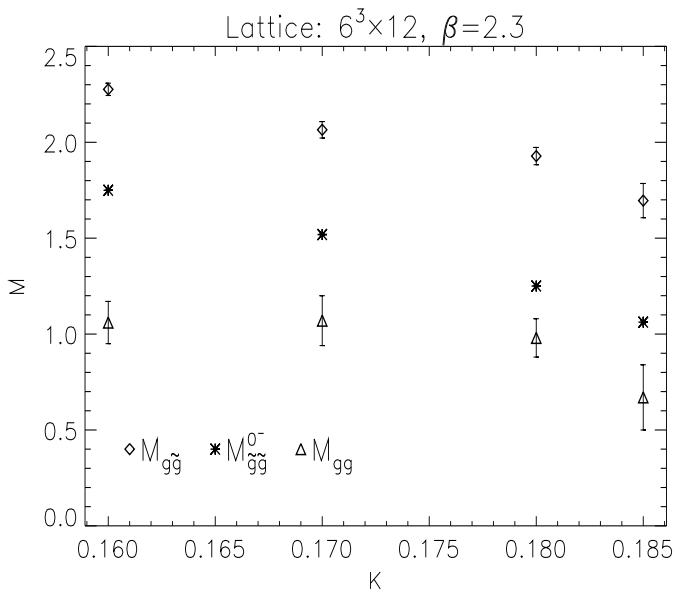

Figure 4. Dependence of the lowest bound state masses on the hopping parameter $K$ on $6^{3} \cdot 12$ lattices at $\beta=2.3$. Masses of states made out of gluons $(g)$ and gluinos $(\tilde{g})$ are shown. (Ref. [24])

\subsection{Other questions}

Besides the phase structure and the low lying mass spectrum there are also other interesting questions which can be studied by Monte Carlo simulations. For instance, the direct investigation of broken supersymmetry Ward-Takahashi identities is possible. In general, the restoration of supersymmetry in the continuum limit has to be understood. Working with gluinos will teach us a lot about the simulation of non-even numbers of flavours and about fermions in the adjoint representation. We shall hopefully get answers to these and other related questions at forthcoming lattice conferences.

\section{REFERENCES}

1. J. Wess, J. Bagger, Supersymmetry and Supergravity, Princeton University Press, Princeton, 1983.

2. P. Fayet, S. Ferrara, Phys. Rep. 32 (1977) 249.

3. M.F. Sohnius, Phys. Rep. 128 (1985) 39.
4. N. Seiberg and E. Witten, Nucl. Phys. B426 (1994) 19; ERRATUM ibid. B430 (1994) 485.

5. D. Amati, K. Konishi, Y. Meurice, G.C. Rossi and G. Veneziano, Phys. Rep. 162 (1988) 169.

6. E. Witten, Nucl. Phys. B202 (1982) 253.

7. G. Curci and G. Veneziano, Nucl. Phys. B292 (1987) 555.

8. S.D.H. Hsu, hep-th/9704149.

9. R. Narayanan, P. Vranas, hep-lat/9702005, hep-lat/9709014 and this Proceedings.

10. J. Nishimura, Phys. Lett. B406 (1997) 215; S. Aoki, K. Nagai, S.V. Zenkin, hep-lat/9705001.

11. P. Huet, R. Narayanan, H. Neuberger, Phys. Lett. B380 (1996) 291.

12. N. Maru, J. Nishimura, hep-th/9705152.

13. S. Gottlieb, W. Liu, D. Toussaint, R.L. Renken, R.L. Sugar, Phys. Rev. D35 (1987) 2531.

14. A. Donini, M. Guagnelli, Phys. Lett. B383 (1996) 301.

15. M. Lüscher, Nucl. Phys. B418 (1994) 637.

16. A.D. Kennedy, J. Kuti, S. Meyer and B.J. Pendleton, Phys. Rev. D38 (1988) 627.

17. I. Montvay, Nucl. Phys. B466 (1996) 259.

18. J. Verbaarschot, hep-th/9709032.

19. I. Montvay, hep-lat/9707005; also see my web page at http://www.desy.de/ montvay.

20. A. Boriçi and Ph. de Forcrand, Nucl. Phys. B454 (1995) 645;

A. Borrelli, P. de Forcrand and A. Galli, Nucl. Phys. B477 (1996) 809.

21. B. Jegerlehner, Nucl. Phys. B53, Proc. Suppl. (1997) 959.

22. G. Koutsoumbas, I. Montvay, Phys. Lett. B398 (1997) 130.

23. A. Donini, M. Guagnelli, P. Hernandez, A. Vladikas, hep-lat/9708006 and this Proceedings.

24. G. Koutsoumbas, I. Montvay, A. Pap, K. Spanderen, D. Talkenberger, J. Westphalen, this Proceedings.

25. G. Veneziano, S. Yankielowicz, Phys. Lett. B113 (1982) 231.

26. A. Kovner, M. Shifman, Phys. Rev. D56 (1997) 2396.

27. A. Masiero, G. Veneziano, Nucl. Phys. B249 (1985) 593; N. Evans, S.D.H. Hsu, M. Schwetz, hep-th/9707260. 